\title{
ON A SEQUENCE INVOLVING SUMS OF PRIMES
}

\author{
ZHI-WEI SUN \\ (Received 2 August 2012; accepted 5 October 2012; first published online 18 January 2013)
}

\begin{abstract}
For $n=1,2,3, \ldots$ let $S_{n}$ be the sum of the first $n$ primes. We mainly show that the sequence $a_{n}=$ $\sqrt[n]{S_{n} / n}(n=1,2,3, \ldots)$ is strictly decreasing, and moreover the sequence $a_{n+1} / a_{n}(n=10,11, \ldots)$ is strictly increasing. We also formulate similar conjectures involving twin primes or partitions of integers.
\end{abstract}

2010 Mathematics subject classification: primary 11A41; secondary 05A17, 05A20, 11B75, 11B83, 11J99, $11 \mathrm{P} 83$.

Keywords and phrases: primes, sums of primes, monotonicity, twin primes, partitions of integers.

\section{Introduction}

For $n \in \mathbb{Z}^{+}=\{1,2,3, \ldots\}$ let $p_{n}$ denote the $n$th prime. The unsolved Firoozbakht conjecture (see [R, p. 185]) asserts that

$$
\sqrt[n]{p_{n}}>\sqrt[n+1]{p_{n+1}} \quad \text { for all } n \in \mathbb{Z}^{+}
$$

that is, the sequence $\left(\sqrt[n]{p_{n}}\right)_{n \geqslant 1}$ is strictly decreasing. This implies the inequality $p_{n+1}-p_{n}<\log ^{2} p_{n}-\log p_{n}+1$ for large $n$, which is even stronger than Cramér's conjecture $p_{n+1}-p_{n}=O\left(\log ^{2} p_{n}\right)$. Let $P_{n}$ be the product of the first $n$ primes. Then $P_{n}<p_{n+1}^{n}$ and hence $P_{n}^{n+1}<P_{n+1}^{n}$. So the sequence $\left(\sqrt[n]{P_{n}}\right)_{n \geqslant 1}$ is strictly increasing.

Now let us look at a simple example not related to primes.

ExAmple 1.1. Let $a_{n}=\sqrt[n]{n}$ for $n \in \mathbb{Z}^{+}$. Then the sequence $\left(a_{n}\right)_{n \geqslant 3}$ is strictly decreasing, and the sequence $\left(a_{n+1} / a_{n}\right)_{n \geqslant 4}$ is strictly increasing. To see this we investigate the function $f(x)=\log \left(x^{1 / x}\right)=(\log x) / x$ with $x \geqslant 3$. As $f^{\prime}(x)=(1-\log x) / x^{2}<0$, we have $f(n)>f(n+1)$ for $n=3,4, \ldots$ Since

$$
f^{\prime \prime}(x)=\frac{2 \log x-3}{x^{3}}>0 \quad \text { for } x \geqslant 4.5,
$$

Supported by the National Natural Science Foundation (grant 11171140) of China and the PAPD of Jiangsu Higher Education Institutions.

(C) 2013 Australian Mathematical Publishing Association Inc. 0004-9727/2013 \$16.00 
the function $f(x)$ is strictly convex over the interval $(4.5,+\infty)$ and so

$$
2 f(n+1)<f(n)+f(n+2)\left(\text { that is, } a_{n+1}^{2}<a_{n} a_{n+2}\right) \quad \text { for } n=5,6, \ldots
$$

The inequality $a_{5}^{2}<a_{4} a_{6}$ can be verified directly.

A sequence $\left(a_{n}\right)_{n \geqslant 1}$ of nonnegative real numbers is said to be log-convex if $a_{n+1}^{2} \leqslant$ $a_{n} a_{n+2}$ for all $n=1,2,3, \ldots$ Many combinatorial sequences (such as the sequence of Catalan numbers) are log-convex; the reader may consult [LW] for some results on log-convex sequences.

For $n \in \mathbb{Z}^{+}$let $S_{n}=\sum_{k=1}^{n} p_{k}$ be the sum of the first $n$ primes. For instance,

$$
S_{1}=2, \quad S_{2}=2+3=5, \quad S_{3}=2+3+5=10, \quad S_{4}=2+3+5+7=17 .
$$

Recently the author [S] conjectured that for any positive integer $n$ the interval $\left(S_{n}, S_{n+1}\right)$ contains a prime. As $S_{n}<n p_{n+1}$ for all $n \in \mathbb{Z}^{+}$, the sequence $\left(S_{n} / n\right)_{n \geqslant 1}$ is strictly increasing.

In the next section we will state our theorems involving the sequence $\left(a_{n}\right)_{n \geqslant 1}$ with $a_{n}=\sqrt[n]{S_{n} / n}$, and pose three related conjectures for further research. Section 3 is devoted to our proofs of the theorems.

\section{Our results and conjectures}

THEOREM 2.1. The sequences $\left(\sqrt[n]{S_{n}}\right)_{n \geqslant 2}$ and $\left(\sqrt[n]{S_{n} / n}\right)_{n \geqslant 1}$ are strictly decreasing.

REMARK 2.2. Note that $S_{n} / n$ is just the arithmetic mean of the first $n$ primes. It is interesting to compare Theorem 2.1 with Firoozbakht's conjecture that $\left(\sqrt[n]{p_{n}}\right)_{n \geqslant 1}$ is strictly decreasing.

For $\alpha>0$ and $n \in \mathbb{Z}^{+}$define

$$
S_{n}^{(\alpha)}=\sum_{k=1}^{n} p_{k}^{\alpha} .
$$

We actually obtain the following extension of Theorem 2.1 .

Theorem 2.3. Let $\alpha \geqslant 1$ and $n \in \mathbb{Z}^{+}$with $n \geqslant \max \left\{100, e^{2 \times 1.348^{\alpha}+1}\right\}$. Then

$$
\sqrt[n]{\frac{S_{n}^{(\alpha)}}{n}}>\sqrt[n+1]{\frac{S_{n+1}^{(\alpha)}}{n+1}}
$$

and hence

$$
\sqrt[n]{S_{n}^{(\alpha)}}>\sqrt[n+1]{S_{n+1}^{(\alpha)}}
$$

Remark 2.4. In view of Example 1.1, (2.1) implies (2.2) if $n \geqslant 3$. We conjecture that (2.1) holds for any $\alpha>0$ and $n \in \mathbb{Z}^{+}$. 
Note that $\left\lfloor e^{2 \times 1.348+1}\right\rfloor=40$ and we can easily verify that

$$
\sqrt[n]{\frac{S_{n}}{n}}>\sqrt[n+1]{\frac{S_{n+1}}{n+1}} \quad \text { for every } n=1, \ldots, 99 .
$$

So Theorem 2.1 follows from Theorem 2.3 in the case $\alpha=1$.

Corollary 2.5. For each $\alpha \in\{2,3,4\}$, the sequences

$$
\left(\sqrt[n]{\frac{S_{n}^{(\alpha)}}{n}}\right)_{n \geqslant 1} \text { and }\left(\sqrt[n]{S_{n}^{(\alpha)}}\right)_{n \geqslant 1}
$$

are strictly decreasing.

Proof. Observe that

$$
\left\lfloor e^{2 \times 1.348^{2}+1}\right\rfloor=102, \quad\left\lfloor e^{2 \times 1.348^{3}+1}\right\rfloor=364, \quad\left\lfloor e^{2 \times 1.348^{4}+1}\right\rfloor=2005 .
$$

In light of Theorem 2.3 and Example 1.1, it suffices to verify that

$$
\sqrt[n]{\frac{S_{n}^{(\alpha)}}{n}}>\sqrt[n+1]{\frac{S_{n+1}^{(2)}}{n+1}}
$$

whenever $\alpha \in\{2,3,4\}$ and $n \in\left\{1, \ldots,\left\lfloor e^{2 \times 1.348^{\alpha}+1}\right\rfloor\right\}$. This can be easily done via computer.

The following theorem is more sophisticated than Theorem 2.3.

Theorem 2.6. Let $\alpha \geqslant 1$. Then the sequence

$$
\left(\sqrt[n+1]{S_{n+1}^{(\alpha)} /(n+1)} / \sqrt[n]{S_{n}^{(\alpha)} / n}\right)_{n \geqslant N(\alpha)}
$$

is strictly increasing, where

$$
N(\alpha)=\max \left\{350000,\left\lceil e^{\left((\alpha+1)^{2} 1.2^{2 \alpha+1}+(\alpha+1) 1.2^{\alpha+1}\right) / \alpha}\right\rceil\right\} .
$$

Corollary 2.7. All the sequences

$$
\begin{aligned}
& \left(\sqrt[n+1]{S_{n+1} /(n+1)} / \sqrt[n]{S_{n} / n}\right)_{n \geqslant 10}, \quad\left(\sqrt[n+1]{S_{n+1}} / \sqrt[n]{S_{n}}\right)_{n \geqslant 5}, \\
& \left(\sqrt[n+1]{S_{n+1}^{(2)} /(n+1)} / \sqrt[n]{S_{n}^{(2)} / n}\right)_{n \geqslant 13}, \quad\left(\sqrt[n+1]{S_{n+1}^{(2)}} / \sqrt[n]{S_{n}^{(2)}}\right)_{n \geqslant 10}, \\
& \left(\sqrt[n+1]{S_{n+1}^{(3)} /(n+1)} / \sqrt[n]{S_{n}^{(3)} / n}\right)_{n \geqslant 17}, \quad\left(\sqrt[n+1]{S_{n+1}^{(3)}} / \sqrt[n]{S_{n}^{(3)}}\right)_{n \geqslant 10}, \\
& \left(\sqrt[n+1]{S_{n+1}^{(4)} /(n+1)} / \sqrt[n]{S_{n}^{(4)} / n}\right)_{n \geqslant 35}, \quad\left(\sqrt[n+1]{S_{n+1}^{(4)}} / \sqrt[n]{S_{n}^{(4)}}\right)_{n \geqslant 17}
\end{aligned}
$$

are strictly increasing. 
Proof. For $N(\alpha)$ given by (2.3), via computation

$$
N(1)=350000, \quad N(2)=974267, \quad N(3)=3163983273
$$

and

$$
N(4)=2271069361863763 .
$$

Via computer we can verify that

$$
\frac{\sqrt[n+1]{S_{n+1}^{(\alpha)} /(n+1)}}{\sqrt[n]{S_{n}^{(\alpha)} / n}}<\frac{\sqrt[n+2]{S_{n+2}^{(\alpha)} /(n+2)}}{\sqrt[n+1]{S_{n+1}^{(\alpha)} /(n+1)}}
$$

for all $\alpha \in\{1,2,3,4\}$ and $n=N_{0}(\alpha), \ldots, N(\alpha)-1$, where

$$
N_{0}(1)=10, \quad N_{0}(2)=13, \quad N_{0}(3)=17, \quad N_{0}(4)=35 .
$$

Combining this with Theorem 2.6, we obtain that

$$
\left(\sqrt[n+1]{S_{n+1} /(n+1)} / \sqrt[n]{S_{n} / n}\right)_{n \geqslant N_{0}(\alpha)}
$$

is strictly increasing for each $\alpha=1,2,3,4$. Recall that $(\sqrt[n+1]{n+1} / \sqrt[n]{n})_{n \geqslant 4}$ is strictly increasing by Example 1.1. So $\left(\sqrt[n+1]{S_{n+1}} / \sqrt[n]{S_{n}}\right)_{n \geqslant N_{0}(\alpha)}$ is strictly increasing for any $\alpha \in\{1,2,3,4\}$. It remains to check that

$$
\frac{\sqrt[n+1]{S_{n+1}^{(\alpha)}}}{\sqrt[n]{S_{n}^{(\alpha)}}}<\frac{\sqrt[n+2]{S_{n+2}^{(\alpha)}}}{\sqrt[n+1]{S_{n+1}^{(\alpha)}}}
$$

for all $\alpha \in\{1,2,3,4\}$ and $n=n_{0}(\alpha), \ldots, N_{0}(\alpha)-1$, where $n_{0}(1)=5, n_{0}(2)=n_{0}(3)=$ 10 , and $n_{0}(4)=17$. This can be easily done via computer.

We conclude this section by posing three conjectures.

Conjecture 2.8. The two constants

$$
s_{1}=\sum_{n=1}^{\infty} \frac{1}{S_{n}} \quad \text { and } \quad s_{2}=\sum_{n=1}^{\infty} \frac{(-1)^{n}}{S_{n}}
$$

are both transcendental numbers.

REMARK 2.9. Our computation shows that $s_{1} \approx 1.023476$ and $s_{2} \approx-0.3624545778$.

If $p$ and $p+2$ are both primes, then they are called twin primes. The famous twin prime conjecture states that there are infinitely many twin primes.

Conjecture 2.10. (i) If $\left\{t_{1}, t_{1}+2\right\}, \ldots,\left\{t_{n}, t_{n}+2\right\}$ are the first $n$ pairs of twin primes, then the first prime $t_{n+1}$ in the next pair of twin primes is smaller than $t_{n}^{1+1 / n}$, that is, $\sqrt[n]{t_{n}}>\sqrt[n+1]{t_{n+1}}$. 
(ii) The sequence $\left(\sqrt[n+1]{T_{n+1}} / \sqrt[n]{T_{n}}\right)_{n \geqslant 9}$ is strictly increasing with limit 1 , where $T_{n}=$ $\sum_{k=1}^{n} t_{k}$

REMARK 2.11. Via Mathematica the author has verified that $\sqrt[n]{t_{n}}>\sqrt[n+1]{t_{n+1}}$ for all $n=$ $1, \ldots, 500000$, and $\sqrt[n+1]{T_{n+1}} / \sqrt[n]{T_{n}}<\sqrt[n+2]{T_{n+2}} / \sqrt[n+1]{T_{n+1}}$ for all $n=9, \ldots, 500000$. Note that $t_{500000}=115438667$.

Recall that a partition of a positive integer $n$ is a way of writing $n$ as a sum of positive integers with the order of addends ignored. Also, a strict partition of $n \in \mathbb{Z}^{+}$ is a way of writing $n$ as a sum of distinct positive integers with the order of addends ignored. For $n=1,2,3, \ldots$ we denote by $p(n)$ and $p_{*}(n)$ the number of partitions of $n$ and the number of strict partitions of $n$ respectively. It is known that

$$
p(n) \sim \frac{e^{\pi \sqrt{2 n / 3}}}{4 \sqrt{3} n} \quad \text { and } \quad p_{*}(n) \sim \frac{e^{\pi \sqrt{n / 3}}}{4\left(3 n^{3}\right)^{1 / 4}} \quad \text { as } n \rightarrow+\infty
$$

(see [HR] and [AS, p. 826]) and hence $\lim _{n \rightarrow \infty} \sqrt[n]{p(n)}=\lim _{n \rightarrow \infty} \sqrt[n]{p_{*}(n)}=1$. Here we formulate a conjecture similar to Conjecture 2.10.

Conjecture 2.12. For $n \in \mathbb{Z}^{+}$let

$$
q(n)=\frac{p(n)}{n}, \quad q_{*}(n)=\frac{p_{*}(n)}{n}, \quad r(n)=\sqrt[n]{q(n)} \quad \text { and } \quad r_{*}(n)=\sqrt[n]{q_{*}(n)} .
$$

Then the sequences $(q(n+1) / q(n))_{n \geqslant 31}$ and $\left(q_{*}(n+1) / q_{*}(n)\right)_{n \geqslant 44}$ are strictly decreasing, and the sequences $(r(n+1) / r(n))_{n \geqslant 60}$ and $\left(r_{*}(n+1) / r_{*}(n)\right)_{n \geqslant 120}$ are strictly increasing.

REMARK 2.13. Via Mathematica we have verified the conjecture for $n$ up to $10^{5}$. In light of Example 1.1, Conjecture 2.12 implies that all the sequences

$$
\left(\frac{p(n+1)}{p(n)}\right)_{n \geqslant 25}, \quad\left(\frac{p_{*}(n+1)}{p_{*}(n)}\right)_{n \geqslant 32}, \quad(\sqrt[n]{p(n)})_{n \geqslant 6}, \quad\left(\sqrt[n]{p_{*}(n)}\right)_{n \geqslant 9}
$$

are strictly decreasing, and that the sequences $(\sqrt[n+1]{p(n+1)} / \sqrt[n]{p(n)})_{n \geqslant 26}$ and $\left(\sqrt[n+1]{p_{*}(n+1)} / \sqrt[n]{p_{*}(n)}\right)_{n \geqslant 45}$ are strictly increasing. The fact that $(p(n+1) / p(n))_{n \geqslant 25}$ is strictly decreasing was conjectured by Chen [C] and proved by Janoski [J, pp. 7-23].

\section{Proofs of Theorems 2.3 and 2.6}

Lemma 3.1. Let $\alpha \geqslant 1$ and $n \in\{2,3, \ldots\}$. Then

$$
S_{n}^{(\alpha)}>2^{\alpha}+\frac{n^{\alpha+1} \log ^{\alpha} n}{\alpha+1}\left(1-\frac{\alpha}{(\alpha+1) \log n}\right) .
$$

Proof. It is known that $p_{k} \geqslant k \log k$ for $k=2,3, \ldots$ (see [Ro] and [RS, (3.12)]). Thus

$$
S_{n}^{(\alpha)}-2^{\alpha}=\sum_{k=2}^{n} p_{k}^{\alpha} \geqslant \sum_{k=2}^{n}(k \log k)^{\alpha}>\sum_{k=2}^{n} \int_{k-1}^{k}(x \log x)^{\alpha} d x=\int_{1}^{n}(x \log x)^{\alpha} d x .
$$


Using integration by parts,

$$
\begin{aligned}
\int_{1}^{n}(x \log x)^{\alpha} d x & =\left.\frac{x^{\alpha+1}}{\alpha+1} \log ^{\alpha} x\right|_{x=1} ^{n}-\int_{1}^{n}\left(\frac{x^{\alpha+1}}{\alpha+1} \cdot \frac{\alpha(\log x)^{\alpha-1}}{x}\right) d x \\
& =\frac{n^{\alpha+1}}{\alpha+1} \log ^{\alpha} n-\frac{\alpha}{\alpha+1} \int_{1}^{n} x^{\alpha}(\log x)^{\alpha-1} d x \\
& \geqslant \frac{n^{\alpha+1}}{\alpha+1} \log ^{\alpha} n-\frac{\alpha}{\alpha+1} \int_{1}^{n} x^{\alpha}(\log n)^{\alpha-1} d x \\
& \geqslant \frac{n^{\alpha+1}}{\alpha+1} \log ^{\alpha} n-\frac{\alpha n^{\alpha+1}}{(\alpha+1)^{2}}(\log n)^{\alpha-1} .
\end{aligned}
$$

Therefore (3.1) holds.

Lemma 3.2. Let $\alpha \geqslant 1$ and $n \in \mathbb{Z}^{+}$with $n \geqslant 55$. Then

$$
\log S_{n}^{(\alpha)}>(\alpha+1) \log n
$$

Proof. Note that $54<e^{4}<55 \leqslant n$. As $\log ^{\alpha} n>4^{\alpha}=\left(2^{\alpha}\right)^{2} \geqslant(\alpha+1)^{2}$, by Lemma 3.1

$$
S_{n}^{(\alpha)}>\frac{n^{\alpha+1} \log ^{\alpha} n}{\alpha+1}\left(1-\frac{\alpha}{\alpha+1}\right)=\frac{n^{\alpha+1}}{(\alpha+1)^{2}} \log ^{\alpha} n \geqslant n^{\alpha+1}
$$

and hence (3.2) follows.

Proof of Theorem 2.3. It is known that

$$
p_{m}<m(\log m+\log \log m)
$$

for any $m \geqslant 6$ (see [RS, (3.13)] and [D, Lemma 1]). If $m \geqslant 101$, then

$$
\frac{\log \log m}{\log m} \leqslant \frac{\log \log 101}{\log 101}<0.3314
$$

and hence $p_{m}<1.3314 m \log m$. As $n+1 \leqslant 1.01 n$,

$$
\frac{\log (n+1)}{\log n}=1+\frac{\log ((n+1) / n)}{\log n} \leqslant 1+\frac{\log 1.01}{\log n} \leqslant 1+\frac{\log 1.01}{\log 100}<1.0022 .
$$

Therefore

$$
p_{n+1}<1.3314(n+1) \log (n+1)<1.3314 \times 1.01 n \times 1.0022 \log n<1.348 n \log n .
$$


Combining Lemmas 3.1 and 3.2, we see that

$$
\begin{aligned}
S_{n}^{(\alpha)} & \left(\frac{n+1}{n^{1+1 / n}} \sqrt[n]{S_{n}^{(\alpha)}}-1\right) \\
& =S_{n}^{(\alpha)}\left(e^{\left(\log S_{n}^{(\alpha)}\right) / n+\log (n+1)-(1+1 / n) \log n}-1\right) \\
& \geqslant S_{n}^{(\alpha)}\left(e^{\left(\log S_{n}^{(\alpha)}-\log n\right) / n}-1\right) \geqslant S_{n}^{(\alpha)}\left(e^{(\alpha \log n) / n}-1\right) \\
& >\frac{n^{\alpha+1} \log ^{\alpha} n}{\alpha+1}\left(1-\frac{\alpha}{(\alpha+1) \log n}\right) \frac{\alpha \log n}{n} \\
& =\frac{\alpha}{\alpha+1}(n \log n)^{\alpha}\left(\log n-\frac{\alpha}{\alpha+1}\right) \\
& >\frac{(n \log n)^{\alpha}}{2}(\log n-1) .
\end{aligned}
$$

As $(\log n-1) / 2 \geqslant 1.348^{\alpha}$, from the above

$$
(n+1)\left(\frac{S_{n}^{(\alpha)}}{n}\right)^{1+1 / n}-S_{n}^{(\alpha)}>(1.348 n \log n)^{\alpha}>p_{n+1}^{\alpha}
$$

and hence

$$
\left(\frac{S_{n}^{(\alpha)}}{n}\right)^{(n+1) / n}>\frac{S_{n+1}^{(\alpha)}}{n+1}
$$

which yields (2.1). As mentioned in Remark 2.4, (2.2) follows from (2.1). This concludes the proof.

Proof of Theorem 2.6. Fix an integer $n \geqslant N(\alpha)$. For any integer $m \geqslant 350001$,

$$
\frac{\log \log m}{\log m} \leqslant \frac{\log \log 350001}{\log 350001}<0.1996
$$

and hence

$$
p_{m}<m(\log m)\left(1+\frac{\log \log m}{\log m}\right)<1.1996 m \log m .
$$

As $n \geqslant 350000$,

$$
\frac{\log (n+1)}{\log n}=1+\frac{\log (1+1 / n)}{\log n} \leqslant \frac{\log 350001}{\log 350000}<1+10^{-6} .
$$

Therefore

$$
\begin{aligned}
p_{n+1} & <1.1996(n+1) \log (n+1) \\
& <1.1996 \times \frac{350001}{350000} n \times\left(1+10^{-6}\right) \log n<1.2 n \log n .
\end{aligned}
$$

Since $\log n \geqslant \log 350000>1 / 0.078335$, Lemma 3.1 implies that

$$
S_{n}^{(\alpha)}>\frac{n^{\alpha+1} \log ^{\alpha} n}{\alpha+1}(1-0.078335)>\frac{n^{\alpha+1} \log ^{\alpha} n}{1.085(\alpha+1)} .
$$


Therefore

$$
q_{n}^{(\alpha)}:=\frac{p_{n+1}^{\alpha}}{S_{n}^{(\alpha)}}<\frac{c_{\alpha}}{n}
$$

where $c_{\alpha}=1.085(\alpha+1) 1.2^{\alpha}$.

By calculus,

$$
x-\frac{x^{2}}{2}<\log (1+x)<x \quad \text { for } x>0
$$

and

$$
-x-x^{2}<\log (1-x)<-x \text { for } 0<x<0.5 \text {. }
$$

Thus

$$
\log \frac{S_{n+1}^{(\alpha)} /(n+1)}{S_{n}^{(\alpha)} / n}=\log \left(1-\frac{1}{n+1}\right)+\log \left(1+q_{n}^{(\alpha)}\right)<-\frac{1}{n+1}+q_{n}^{(\alpha)}
$$

and

$$
\begin{aligned}
\log \frac{S_{n+2}^{(\alpha)} /(n+2)}{S_{n}^{(\alpha)} / n} & >\log \left(1-\frac{2}{n+2}\right)+\log \left(1+2 q_{n}^{(\alpha)}\right) \\
& >-\frac{2}{n+2}-\frac{4}{(n+2)^{2}}+2 q_{n}^{(\alpha)}-2\left(q_{n}^{(\alpha)}\right)^{2}
\end{aligned}
$$

Hence

$$
\begin{aligned}
D_{n}^{(\alpha)} & :=\frac{2}{n+1} \log \frac{S_{n+1}^{(\alpha)}}{n+1}-\frac{1}{n} \log \frac{S_{n}^{(\alpha)}}{n}-\frac{1}{n+2} \log \frac{S_{n+2}^{(\alpha)}}{n+2} \\
< & \frac{2}{n+1}\left(\log \frac{S_{n}^{(\alpha)}}{n}-\frac{1}{n+1}+q_{n}^{(\alpha)}\right)-\frac{1}{n} \log \frac{S_{n}^{(\alpha)}}{n} \\
& -\frac{1}{n+2}\left(\log \frac{S_{n}^{(\alpha)}}{n}-\frac{2}{n+2}-\frac{4}{(n+2)^{2}}+2 q_{n}^{(\alpha)}-2\left(q_{n}^{(\alpha)}\right)^{2}\right) \\
= & \frac{-2 \log \left(S_{n}^{(\alpha)} / n\right)}{n(n+1)(n+2)}-\frac{2}{(n+1)^{2}}+\frac{2}{(n+2)^{2}}+\frac{4}{(n+2)^{3}}+\frac{2 q_{n}^{(\alpha)}}{(n+1)(n+2)}+\frac{2\left(q_{n}^{(\alpha)}\right)^{2}}{n+2} .
\end{aligned}
$$

Combining this with (3.2) and (3.3) and noting that (350001/350000) $n^{2} \geqslant n(n+1)$,

$$
\begin{aligned}
D_{n}^{(\alpha)}< & \frac{-2 \alpha \log n}{n(n+1)(n+2)}-\frac{2(2 n+3)}{(n+1)^{2}(n+2)^{2}}+\frac{4}{(n+2)^{3}} \\
& +\frac{2 c_{\alpha}}{n(n+1)(n+2)}+\frac{2 c_{\alpha}^{2}}{n^{2}(n+2)} \\
< & \frac{-2 \alpha \log n}{n(n+1)(n+2)}-\frac{4}{(n+1)(n+2)^{2}}+\frac{4}{(n+1)(n+2)^{2}} \\
& \quad+\frac{2 c_{\alpha}+2(350001 / 350000) c_{\alpha}^{2}}{n(n+1)(n+2)} \\
= & \frac{2\left((350001 / 350000) c_{\alpha}^{2}+c_{\alpha}-\alpha \log n\right)}{n(n+1)(n+2)} .
\end{aligned}
$$


Note that

$$
\begin{aligned}
\frac{350001}{350000} c_{\alpha}^{2}+c_{\alpha} & =\frac{350001}{350000} \times 1.085^{2}(\alpha+1)^{2} 1.2^{2 \alpha}+1.085(\alpha+1) 1.2^{\alpha} \\
& <1.2(\alpha+1)^{2} 1.2^{2 \alpha}+1.2(\alpha+1) 1.2^{\alpha} \leqslant \alpha \log N(\alpha) \leqslant \alpha \log n .
\end{aligned}
$$

So we have $D_{n}^{(\alpha)}<0$ and hence

$$
\frac{\sqrt[n+1]{S_{n+1}^{(\alpha)} /(n+1)}}{\sqrt[n]{S_{n}^{(\alpha)} / n}}<\frac{\sqrt[n+2]{S_{n+2}^{(\alpha)} /(n+2)}}{\sqrt[n+1]{S_{n+1}^{(\alpha)} /(n+1)}}
$$

as desired.

\section{Acknowledgement}

This work was done during the author's visit to the University of Illinois at UrbanaChampaign, and the author wishes to thank Professor Bruce Berndt for his kind invitation and hospitality.

\section{References}

[AS] M. Abramowitz and I. A. Stegun (eds.) Handbook of Mathematical Functions with Formulas, Graphs, and Mathematical Tables, 9th printing (Dover, New York, 1972).

[C] W. Y. C. Chen, Recent developments on log-concavity and $q$-log-concavity of combinatorial polynomials. Paper presented at the 22nd Int. Conf. on Formal Power Series and Algebraic Combin. (San Francisco, 2010).

[D] P. Dusart, 'The $k$ th prime is greater than $k(\log k+\log \log k-1)$ for $k \geqslant 2$ ', Math. Comp. 68 (1999), 411-415.

[HR] G. H. Hardy and S. Ramanujan, 'Asymptotic formulae in combinatorial analysis', Proc. Lond. Math. Soc. 17 (1918), 75-115.

[J] J. E. Janoski, A Collection of Problems in Combinatorics, PhD Thesis, Clemson University, May 2012.

[LW] L. L. Liu and Y. Wang, 'On the log-convexity of combinatorial sequences', Adv. in Appl. Math. 39 (2007), 453-476.

[R] P. Ribenboim, The Little Book of Bigger Primes, 2nd edn (Springer, New York, 2004).

[Ro] J. B. Rosser, 'The $n$th prime is greater than $n \log n$ ', Proc. Lond. Math. Soc. 45 (1939), 21-44.

[RS] J. B. Rosser and L. Schoenfeld, 'Approximate formulas for some functions of prime numbers', Illinois J. Math. 6 (1962), 64-94.

[S] Z.-W. Sun, 'On functions taking only prime values', Preprint, 2012, arXiv:1202.6589.

\section{ZHI-WEI SUN, Department of Mathematics, Nanjing University,}

Nanjing 210093, PR China

e-mail: zwsun@nju.edu.cn 